\title{
Editorial
}

\section{Marketing for growth}

Simon Gulliford, who co-authored this editorial with Jan Hall, is the Brand Development Director of Sears PLC and an active consultant in marketing for many other blue chip organisations.

\section{INTRODUCTION}

In the future, the most successful marketers will be those who instigate new trends, rather than following those established by competitors. Playing 'catch-up' is no longer a sustainable option. The swift interpretation of data available from a myriad of sources allied to divergent thinking will be required if activity is to be executed with precision and panache.

Customers are becoming over-choiced and, as a consequence, they are having to become smarter. They are, therefore, becoming frustrated and are understandably too busy to discern fine distinctions that do not deliver enough value to merit the increased effort required to select and ultimately purchase.

If there is a genuine innovation that makes the offer slot naturally into their lives, they will buy it - just so long as the benefits available are both compelling and relevant. Disappointingly, marketers tend to fight for the 'safe ground' and appear committed to dealing in similarities without focusing sufficiently on real salient differences. This, in part, is due to the inability fully to appreciate that many markets are becoming increasingly polarised between those consumers who are income rich and time poor and those who are time rich and income poor.

Both groups are seeking out organisations that have greater empathy with their lifestyles, concerns and purchasing patterns.
In this editorial we consider such issues and outline a new marketing framework that has been specifically created to help consider how growth opportunities can be beneficially exploited.

\section{THESIS - THE OLD ORDER}

The post-war period has been largely characterised by periods of sustained improvement in standards of living. Growth in consumer spending was fuelled by pay settlements ahead of inflation and, in many instances, the greatest challenge for many businesses was to service latent demand. The principle pressure, therefore, was to ensure that a particular business corralled a greater share of growth than its competitors. In such a business environment Fast Moving Consumer Goods (FMCG) flourished, the disciplined science of marketing crystallised and the rules and the techniques of best practice emerged. Sadly, recent times have not conformed to these norms and, increasingly, businesses that merely rely upon the ability to float upwards on 'pre-formed business thermals' are essentially crisis bound.

Many 'fast moving' consumer goods are in danger of being re-christened 'slow changing' consumer goods. In the next decade, latent growth can no longer be taken for granted. Of course, growth still occurs and new niches provide considerable scope and opportunity for genuine creative approaches. However, businesses must become smarter at establishing the extent to which a brand can be stretched and extended without causing real damage to the original proposition. The real debate is about ensuring that the most is being made of 
the brands' core values and that growth is being achieved via optimum focus, rather than convenient promotional short-cuts which potentially 'distress' the brand.

Stepping outside core brand values will either immediately, or over time, dilute the brand's point of view and, in all likelihood, detrimentally weaken both current and future brand extensions.

Take Bulmers, for example. Their Strongbow brand essentially defined the volume cider segment. Outstanding communication and a commitment to exceptional product quality allowed them to command an enviable position in a market that has experienced considerable change. Strongbow's personality and core values were strongly linked to 'hard men, hard drinking' - the personification of a real man's drink. Successive campaigns have reinforced this message, while, at the same time, providing a subtle update to cope with the requirement of the emerging generation of drinkers.
The 1980s saw an emergence of a requirement for low alcohol beverages. Notable successes in this area included Kalibur and Barbican. There now existed irrefutable evidence that sufficient demand existed to justify the launch of a 'low alcohol cider'. Instead of developing a new brand, Bulmers sought to extend the Strongbow brand and hence the launch of Strongbow Low Alcohol.

This is the equivalent of putting a diesel engine in a Ferrari! People drink Strongbow because of the speed at which they will become intoxicated. This simply does not translate to a 'low alcohol' proposition. It has, moreover, created unnecessary confusion in the mind of the customer. It is interesting that this activity coincided with the launch of premium priced, exceptionallystrong bottled cider. It could be argued that Strongbow's dilution provided an opportunity for a proposition such as White Diamond to occupy the strong cider platform previously owned by Strongbow.

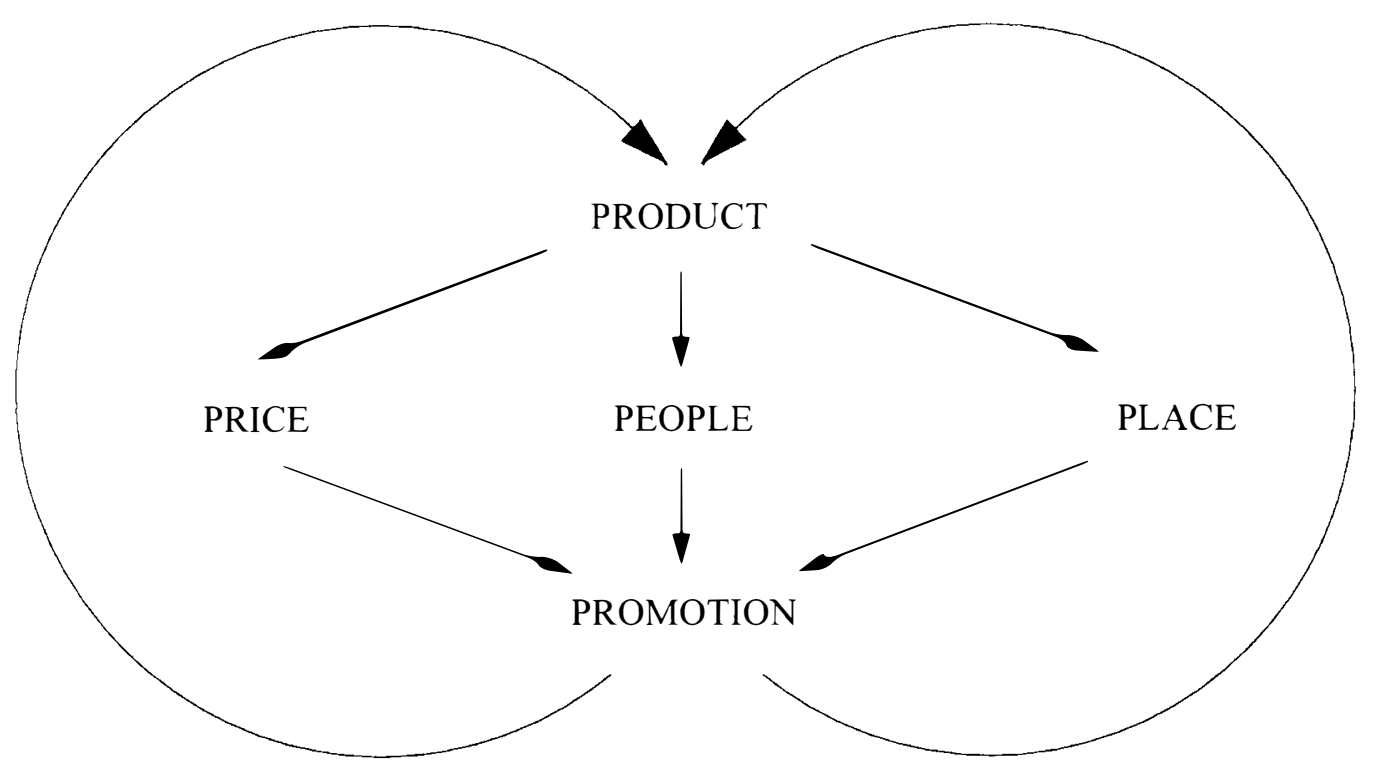

Figure 1

The Five Ps model 
Stretching a brand to the point where the message and the core value becomes a marginalised benefit, leaves the consumer confused and thirty years of brand building severely compromised.

The classic and most rudimentary of marketing models - The Four Ps - Product, Place, Price and Promotion was borne out of FMCG thinking, when top quality marketing represented such companies' trump card. They defined the subject and, as a consequence, nurtured it and aided its migration into other sectors. We have also seen the addition of a fifth $\mathrm{P}$ for People as retail and service companies embraced this traditionally FMCG marketing technique (see Figure 1). The Five Ps are still a vital prerequisite to being 'in the game' in the second half of the 90 s but their limitations are becoming all too obvious. A great deal more is needed.

Top performers that yesterday set the rules have watched others break the rules and take the lead.

\section{ANTITHESIS - THE PARADIGM SHIFTS}

The rules of success are more fluid now than ever before. The consumer is increasingly individualistic and difficult to satisfy.

The IT revolution, media diversification and the proliferation of television channels has led to a desegregated audience. Trend influences and culture credibilities have led to a polarisation within consumer sub-cultures and there is less and less glue to hold those groups together. It is brands that will provide the anchor points to an even greater extent in the future. However, the way in which brands need to behave is evolving rapidly.

For example, for 16 to 25-year olds, Calvin Klein makes a real statement, a kind of bonding for that age group. A mainstream, prime-time television campaign would be irrelevant and possibly even detri- mental because it could not communicate with the appropriate audience/opinion formers. It would also dilute credibility by association if the product appeared between wall-to-wall middle-market products. But advertising positioned on sites outside the clubs at which this age group spend four nights a week, would build a relationship at the right level.

This will not reduce the need to invest in basic brand advertising. Persil will still have to wash whiter than white. Fairy Liquid must always be soft on your hands yet still tough on grease. But today much more is needed for all brands.

\section{SYNTHESIS - A NEW MODEL FOR GROWTH}

It is clear from the continued success of companies such as Ford, Kellogg's and Sainsbury's, which led the way in the past, that it is still critical to be getting the Five Ps right. But to achieve exceptional growth a new game needs to be added. While the complex manoeuvring goes on with the classical game, a second must be played at the same time, on another level.

Consequently, in addition to the Five Ps, we propose a second dimension game with Five Ls. These are:

\section{- Lifestyle; \\ - Leverage; \\ - Location; \\ - Linkage; \\ - Loyalty.}

These create a new agenda for growth (see Figure 2).

The market and the forces that act on it are, of course, a complex matrix of ever shifting values and desires, which is why a clear and simple strategy is required to sustain a benchmark on the growth curve. Brands that will prosper are those that can build themselves into our lives by asking 


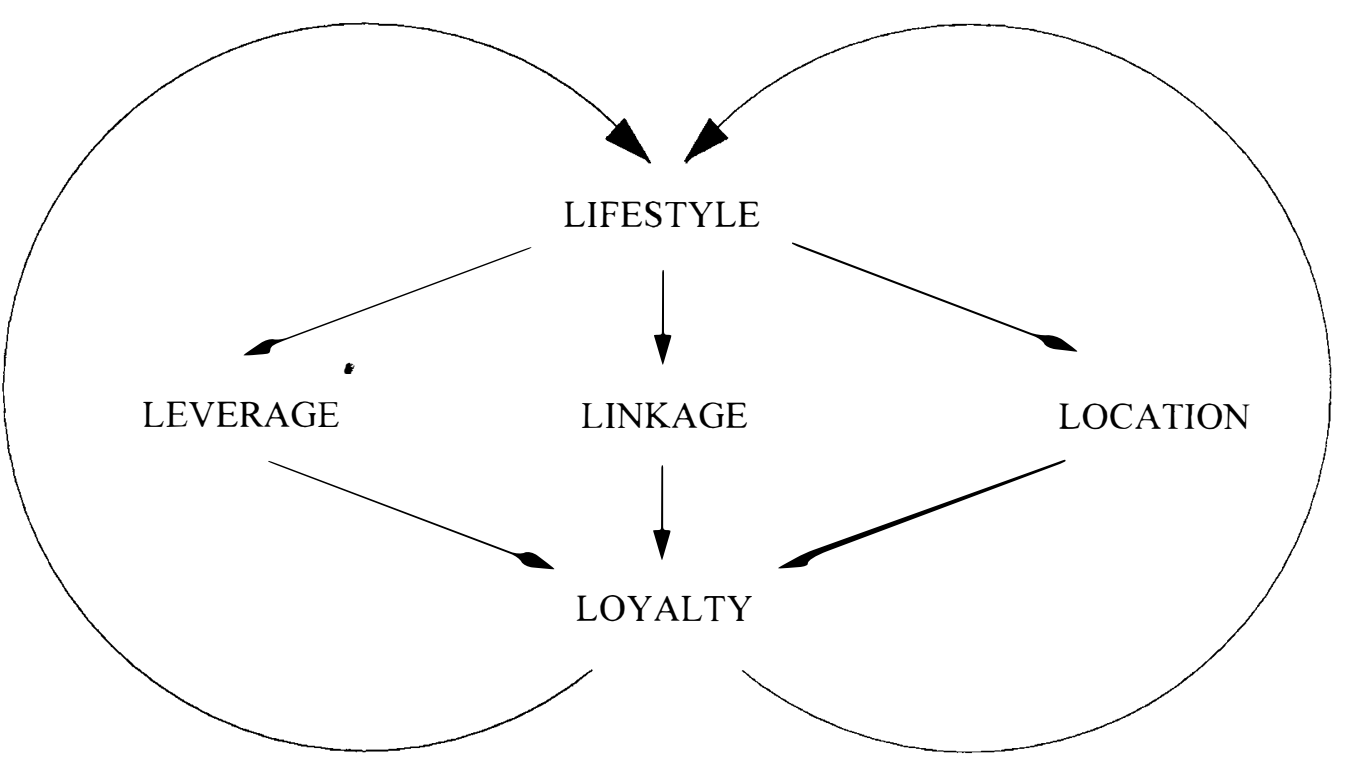

Figure 2

The Five Ls model

how we live, what are the frustrations we face and how the product can answer that need.

\section{Lifestyle}

There are still a surprising number of companies that are failing to think like consumers and get inside their heads in order better to understand their lifestyle, and hence, accurately tailor their products and services to meet their daily problems.

However, some are doing this and doing it very well. Below are some slices of life in the 1990s:

- You get off a 'redeye' flight from a brilliantly successful meeting. You need to get to the office straight away — thank goodness for British Airways. BA has saved you a couple of precious hours by installing showering facilities at their terminals allowing you to go straight from the airport to the office - with no personal comments on arrival.
- MacDonalds produced a classic with their prime-time advertisement showing a father, obviously divorced and only getting to see the kids on Saturday. What does he do with his kids when it's wet and miserable? They'll be bored. He will feel he's failed them - again. But ... he knows where there is a guaranteed chunk of happiness. He knows how to make them feel going out with dad is cool. He takes them to MacDonalds. Happiness is Happy Meals all round. Problem solved.

- It's bath time - mum has her hands full with baby - her other toddler is creating mayhem and her patience is fraying. The little one has really dry skin and she has been advised to apply baby oil when she is cradling the baby after bathing. She used to struggle to keep one eye on the nipper and the other on the infant, while opening the slippy screw top on the bottle and, at the same time, ensuring that it is not knocked over by a 
'Power Ranger' look-alike three-year old. Now she is able to buy baby oil from Boots in a wide based bottle to prevent tipping and a flip top which can be operated with a single hand.

This understanding led to a modified design brief and outstanding new packaging, geared specifically to help a set of lifestyle circumstances. As a result, stress and frustration eased and a platform for real loyalty established.

- Christmas arrives but the Joneses couldn't afford a Play Station for their son unless their mortgage allowed them to miss December's payment.

These examples show clear recognition of consumers' frustrations - and provide practical solutions. Recognise a problem. Deal with it. Take a long hard look at lifestyle and provide the only known antidote to competitive alternatives - prejudice. Create a preference for a particular product by getting it right.

\section{Location}

Location used to mean which shop or merchandising point. Now it is about how the customer chooses to transact. Customers are getting clever, they are habitual innovators. As technology emerges, they find the best application. Consider the denim market. Brand leader Levi's correctly identified that 'fit' was an all important driver of satisfaction. Accordingly, they developed processes and systems to enable measurements to be taken and perfectly-fitting pairs of jeans could then be made to order, available for despatch within two weeks - brilliant custom-made jeans. It was no longer necessary to sample prior to purchase. The customer simply relies on the integrity of the brand to deliver. Young people have less anxiety regarding use of new technology and accordingly they have embraced the potential offered by the Internet. In so doing they have worked out how to get great value.
Levi's are somewhat dismayed to discover Internet surfers down loading their vital statistics, together with credit card details to US websites, where orders placed for custom made Levi's are duly despatched to the UK within three weeks, with a significant cost saving, even when postage and packaging are taken into account.

At last, competitors are waking up to the fact that Argos is actually a player in the home shopping sector. Increasingly, people make their decisions at home then simply pick up their choices from a retail outlet.

\section{Leveraging}

Leveraging is about making the most of the organisation's special capabilities. It is about stretching the brand appropriately, while keeping within its proposition's core value. Consider the example of The Automobile Association. Few organisations would have the guts to fund a campaign which celebrates and highlights the fact that the organisation is in fourth position. Being the fourth emergency services is a brilliant concept. Why? Because positions one, two and three are occupied and are essentially never going to be available. Being fourth means that the very best their competitors can hope to be is fifth.

How does this link to leverage? First look at what capability potentially makes the AA special. Undoubtedly they have an enviable skill of finding people in distress in potentially remote locations. They have achieved this through years of experience and massive investment in information technology and communication systems. Where else might this skill be leveraged together with the brand values associated with the AA? Imagine in five years time pressure upon the Government further to reduce the costs associated with running the NHS. Think what other services could be sub-contracted, indeed privatised. Ambulance services must be quite high on the list. What capability is required to operate an ambu- 
lance service? Finding people in distress in potentially remote locations? Imaginary but not impossible, perhaps it is just as well the AA have prepared us to think of them as an integral part of the emergency service network. How would a customer rather be picked up? By an AA ambulance or a Group 4 ambulance?

Other clever examples are $M$ \& $S$ and Richard Branson moving into financial services. People trust them although for slightly different reasons. Customers trust $M \& S$ because it stands for quality and value for money - and they feel that there is less chance of being overcharged. They trust Branson because they see him as people's champion who they believe will deliver something that is reasonably straight.

\section{Linkage}

Linkage is another L, rare in the past, but gaining increasing currency. This technique acknowledges particular qualities of the brand, that may be further enhanced through linkages with organisations seeking to gain a deeper and more comprehensive relationship with the customer.

Texaco's 'Children Should be Seen and Not Hurt' campaign is a good example of a community involvement linkage. Texaco approached the Department of Transport with a plan to back their campaign to reduce the number of children being killed and injured by motorists. The campaign's success raised the company's profile and helped improve business performance.

Finding the right links is the key. Sears Group achieved outstanding results when Miss Selfridge was matched with Ariel washing powder.

Ariel is the leading brand of detergent in the UK. It has maintained this position by a range of significant improvements in product performance. However, its communication techniques have remained largely traditional and wedded to tried and tested approaches that emphasise a series of pre- dominantly rational reasons for purchasing Ariel. Miss Selfridge, on the other hand, operates in an extremely volatile segment of the UK fashion market and has to deal with teenage customers who, by their very nature, are transient and likely to trade up when they find themselves in full-time employment. In contrast to buying Ariel, making purchases at Miss Selfridge is a highly emotionally charged process; conforming to latest fashion, while feeling confident about their own appearance is an extremely difficult trick for young women to achieve.

Linking both brands resulted in considerable benefit for both. Ariel's new Futur formulation meant that customers' clothes looked good for longer. As a consequence, customers could have a larger selection of clothes to choose from, as their wardrobe would not suffer from the natural attrition associated with clothes fading and losing their initial lustre. Moreover, the implicit trust that is associated with Ariel helped to create a halo effect for Miss Selfridge, while Miss Selfridge, in turn, added considerable credibility to the notion that Ariel was in tune with the lives and fashion requirements of young women.

This was further extended through press activity, where More Magazine (the EMAP youth title) was selected as a vehicle to promote a competition, sponsored by Ariel and managed by Miss Selfridge, in which clothes were designed by readers. The best clothes would then be manufactured and sold to the Miss Selfridge outlets with an element of the proceeds donated to a charity selected by Miss Selfridge staff. Again, all participants mutually benefited.

\section{Loyalty}

The last $\mathrm{L}$ is Loyalty. It is a move away from the one-off hit and involves building relationships with customers and the desired brand position for the future.

Shell's Smart campaign is an excellent illustration of this fifth $\mathrm{L}$. 
Every year nearly every one of Britain's 24 million motorists uses Shell at some point. But it also faces strident competition from other suppliers, including major brands and supermarkets which have built a significant share of the fuel market in ten years.

The companies' objectives were to increase its market share by returning to a long-term, strategic loyalty scheme. It had to offer ease of collection, attractive rewards for target consumer segments, preferential appeal and provide a mechanism for building a dialogue with its customers. It would capitalise on network strength and brand value.

It built the relationship by: identifying a need to combine long-term 'collectability' with short-term tactical appeal; using different promotional techniques for different sectors; building on market success with partner retailers; creating a direct dialogue between Shell and its customers and using technology to enhance appeal.

The benefits are prodigious. Smart Card enabled customer purchasing records to be built up, yielding a database of every promotionally active customer. It also supplies that vital channel of communication between company and customer providing purchase data, weight and regularity of purchase, customer details and the ability to promote directly to key target audiences.

The Smart Relationship provides benefits both ways. Customers get long-term reward opportunities and short-term changing offers on the forecourt including air miles; direct redemption at UCI Cinemas and First Leisure, charity donations, instant redemption items; home-delivered gifts and a regular catalogue featuring new offers.

Shell gets a genuine added benefit to the brand; long-term incremental sales to existing customers and crossovers from rival brands. The future will see Smart develop multi-collective redemption through partners, like John Menzies, growth of the database through more participants, driving valued customer traffic to partners, quicker access to rewards and a longer-term move from catalogues to direct redemption.

\section{THE FIVE LS}

Taken together, the Five Ls can lead to superior existing and future growth potential. A company that demonstrates this clearly is Tesco. In the 1980s and 1990s they not only improved their Five Ps, but they have also been very successful in employing the Five Ls. For each of the Five Ls examples include:

- Lifestyle Tesco Metro on the high street acknowledges the need for top-up shopping for many working people and also acknowledges that over $30 \%$ of the UK population now comprises single households who purchase food on a more frequent basis.

- Leverage Tesco's current account, Clubcard Plus, cleverly exploits their relationship with their customers and brings them an extra service with extra value and extra convenience.

- Linkage Computers for Schools links Tesco to their local communities and further enhances their bond with their customers.

- Location Tesco petrol stations in nonTesco sites gives Tesco greater reach and more brand presence.

- Loyalty Tesco's Club Card has been hugely successful in creating a database of customers that Tesco will go on to exploit in the coming years and crucially has avoided the tendency simply to bribe their customers.

The above Ls, like the traditional Ps, are all inter-linked and each reinforces the other, all around the Tesco core brand values. This is of course essential. As growth becomes ever harder to achieve, the importance of reinforcing core brand values in every action is critical. 


\section{CONCLUSION}

The real power of the Five Ls is that they are actually not focused solely in the marketing department. They are not about vertical marketing department thinking. They are about thinking outside those classic roles, working with the consumer more holistically, maximising internal capabilities and exploiting links with external partners including suppliers and distributors.

The Five Ls invite a customer focus on managing brands, encouraging a move away from production-orientated thinking to functional, process thinking and making innovative but appropriate links both internally and externally. The blueprint for success 'to infinity and beyond' lies in a clear understanding of the brand's core values, understanding consumers' lifestyle and astutely applying that data to find the most effective locations, linkages and leveraging that should all, in turn, create a platform on which loyalty can be built.

Simon Gulliford, Sears PLC and Jan Hall, Editorial Board 
